\title{
Calculable corrections to brane black hole decay. II. Greybody factors for spin 1/2 and 1
}

\author{
Panagiota Kanti \\ Theory Division, CERN, CH-1211 Geneva 23, Switzerland \\ John March-Russell \\ Theory Division, CERN, CH-1211 Geneva 23, Switzerland \\ and Theoretical Physics, Oxford University, 1 Keble Road, OX1 3NP, United Kingdom
}

(Received 20 December 2002; revised manuscript received 21 March 2003; published 22 May 2003)

\begin{abstract}
The production of black holes in extra-dimensional brane-world theories can lead to detectable signals via the Hawking evaporation of the black hole to brane-localized standard model modes. We calculate, as a function of partial wave number and number of toroidally compactified extra dimensions, the leading correction to the energy spectrum of such Hawking radiation (the greybody factors) for decay into spin-1/2 fermions and spin-1 gauge fields localized on the standard model brane. We derive the associated improved differential emission rates for both types of fields. We provide both simple expressions for the leading behavior of the greybody factors in the low-energy limit $\omega r_{H} \ll 1$ and numerical evaluation of our full analytic expressions for the emission rates, valid for $\omega r_{H} \sim 1$. The full analytic expressions demonstrate that both the greybody factors and emission rates are enhanced as the number of extra dimensions increases.
\end{abstract}

DOI: 10.1103/PhysRevD.67.104019

PACS number(s): 04.70.Dy, 04.50.+h, 11.10.Kk

\section{INTRODUCTION}

One of the most spectacular consequences of brane-world theories $[1,2,3]$ with a low fundamental gravitational scale $M_{*} \gtrsim 1 \mathrm{TeV}$ (for some works on the structure of these theories, on their cosmological implications and experimental signatures, see [4]) is the modification of the properties of small black holes [5] that, in principle, allows them to be copiously produced at the CERN Large Hadron Collider (LHC) and in other high-energy processes $[6,7,8,9,10,11,12]$. In a collider setting, the dominant signal for such black hole production arises from the Hawking evaporation products of the black hole, in particular the characteristic spectrum of such radiation. In a previous paper [13], we considered the leading semiclassical corrections to the spectrum of Hawking radiation for a higher-dimensional black hole evaporating to scalar modes. We considered both a scalar field localized to the brane and the situation in which it could propagate in the full $(4+n)$-dimensional bulk and computed the corresponding greybody factors as functions of energy $\omega$, angular momentum $j$, and number of (flat) extra dimensions, $n$. The scalar case is both a useful testing place of the methods used in such a computation and potentially relevant for Higgs, or other scalar, boson production from black holes formed in collisions at the LHC or other high-energy colliders. However, from a practical point of view the most important cases to consider are those of the production of standard model (SM) fermions or SM gauge bosons, to which we turn in this paper.

In both cases the fields that need to be considered are brane localized. For fermions this is because of the chiral nature of the SM fermions, since a fermion propagating in the bulk is necessarily nonchiral from the $4 \mathrm{D}$ perspec- tive. ${ }^{1}$ It is possible to consider SM gauge fields propagating in the higher-dimensional bulk or a subspace thereof [15]. However, the already existing collider constraints bound the size of such gauge extra dimensions to $R_{\text {gauge }}^{-1} \geq 3 \mathrm{TeV}$, while in the best possible case where only the graviton propagates in the extra dimensions, the limits on the new fundamental higher-dimensional Planck scale are of order $M_{*} \geq 1.2 \mathrm{TeV}$ [16]. On the other hand, the validity of the semiclassical description of black holes requires that their horizon radius $r_{H}$ be large compared to the higher-dimensional Planck length $r_{H} \gg 1 / M_{*}$, which given the just cited bounds, as well as the limits on the maximum mass of black hole that can be produced at the LHC, implies that $r_{H}$ must be somewhat large compared to $R_{\text {gauge }}$, too. In this case the gauge fields can also be considered as effectively brane localized, the "brane" in question having some nontrivial substructure. To leading order this substructure does not change the greybody factors we compute for the spin- 1 case.

Note that the properties of a black hole, in particular its Schwarzschild radius $r_{H}$ and production cross section, differ from the $4 \mathrm{D}$ result provided that $r_{H}$ is smaller than the size of at least some of the extra dimensions, $r_{H}<R$. If the brane tension does not strongly perturb the $(4+n)$-dimensional

\footnotetext{
${ }^{1}$ The right handed (RHD) neutrino can propagate in the bulk, leading to an alternate, nonseesaw explanation of the lightness of the observed neutrino states [14]. Thus in principle it is possible to consider the Hawking decay of a black hole to these bulk neutrino states. However, from the perspective of the brane observer this leads only to a missing energy signal which is not distinguishable from the many other sources of missing energy in brane-world theories. Therefore we do not consider this possibility in the present work.
} 
black hole solution and the extra dimensional space is approximately flat on the scale $r_{H}$, then the black hole is well described as an angularly symmetric $(4+n)$-dimensional black hole centered on the brane, but extending out into the $n$ extra dimensions, leading to the expression $[5,17]$

$$
r_{H}=\frac{1}{M_{*}}\left(\frac{M}{M_{*}}\right)^{1 /(n+1)}\left(\frac{8 \Gamma((n+3) / 2)}{(n+2) \pi^{(n+1) / 2}}\right)^{1 /(n+1)}
$$

for the Schwarzschild radius. Here we follow these commonly made assumptions, which have the consequence of limiting our discussion to the Hawking decay of black holes in the context of Arkani-Hamed-Dimopoulos-Dvali-like theories [1]. In particular, black holes in theories of the Randall-Sundrum (RS) type [3] require a more involved treatment (for a recent paper considering the decay of RS black holes see [18]).

After such black holes are produced, they decay by the emission of Hawking radiation, and it is expected that they will decay mostly to particles on our brane [19]. The spectrum of radiation, with a temperature $T_{\mathrm{BH}}=(1+n) /\left(4 \pi r_{H}\right)$ (in $G=k_{B}=c=\hbar=1$ units), is given by the Hawking formula [20]

$$
\frac{d E(\omega)}{d t}=\sum_{j, b} \sigma_{j, b}(\omega) \frac{\omega}{\exp \left(\omega / T_{\mathrm{BH}}\right) \mp 1} \frac{d^{n+3} k}{(2 \pi)^{n+3}},
$$

where $j$ labels the total angular momentum quantum number, $b$ labels any other quantum numbers of the emitted particle, as well as the particle type, and in the phase-space integral $|k|=\omega$ for a massless particle. Here $\sigma(\omega)$, known as the "greybody factor," is an energy-dependent function arising from the backscattering of part of the outgoing radiation back into the black hole by the nontrivial metric in the region exterior to the black hole horizon (for early works on the emission spectra of 4D black holes, see [21]).

In this paper we calculate the greybody factors and emission rates for spin- $1 / 2$ and spin- 1 particles localized to the SM brane in the presence of a nonrotating black hole of the type described above. It is expected that such a nonrotating black hole is a good approximation to the exact solution after the initial "balding" and "spin-down" phases [7]. As discussed for the scalar case in our first paper [13], the greybody factor is computed using its equality with the absorption cross section for the appropriate type of particle incident on the background metric that describes the brane black hole. The equality of greybody factors to absorption cross sections implies that the greybody factors do not invalidate the thermal nature of the black hole.

Finally, it is important to recall that the semiclassical calculation of Hawking emission is only reliable when the energy of the emitted particle is small compared to the black hole mass, $\omega \ll M$, since only in this case is it correct to neglect the back reaction of the metric during the emission process. This in turn requires that the Hawking temperature obey the relation $T_{\mathrm{BH}} \ll M$, which is equivalent to demanding that the black hole mass $M \gg M_{*}$. Inevitably, this condition breaks down during the final stages of the decay process, but for those black holes of initial mass larger than $M_{*}$ most of the evaporation process is within the semiclassical regime.

In Sec. II of this paper, we present the general equation for the radial part of the wave function of fields with spin $1 / 2$ and 1 , as well as a discussion of the metric used in calculating the greybody factors. We proceed to solve this equation at the asymptotic regimes of the near-horizon and far-field zones, for an arbitrary number of extra dimensions and angular momentum number, and we match them in an intermediate zone in order to construct a complete, smooth solution over the whole radial regime.

In Sec. III, the greybody factors for fermionic and gauge fields are computed. Our full analytic results are contained in Eqs. (35)-(38), (40), (46), and (49). Although analytically complicated, these full results are necessary for the accurate determination of the Hawking emission spectrum from brane black holes (see Sec. IV). We expect they will be of use to those searching for black holes at current and future colliders or in ultrahigh-energy cosmic-ray collisions. However, for ease of comparison with previous studies of greybody factors we derive simplified expressions for the leading corrections to the spectrum of the Hawking radiation valid for $\omega r_{H} \ll 1$. These expressions are presented in Eq. (47) for spin $1 / 2$ and Eq. (55) for spin 1, for arbitrary $j$ and $n$. Their values for $n$ $=2,4,6$ extra dimensions and for the first three, and thus most important, angular momentum modes (with $j=\frac{1}{2}, \frac{3}{2}, \frac{5}{2}$ for spin $1 / 2$ and $j=1,2,3$ for spin 1) are tabulated in Tables I and II, respectively.

In Sec. IV, we proceed to calculate the full greybody factors and emission rates for spin-1/2 and spin- 1 fields by a numerical evaluation of our full analytic results of Secs. II and III (valid beyond the simplified limit $\omega r_{H} \ll 1$ ). A similar analysis for spin-0 fields, based on the results derived in [13], is also included in this section. The results are presented in Figs. 1, 2, and 3. Our conclusions are summarized in Sec. V.

\section{GENERAL EQUATION FOR SPIN-1/2 AND -1 FIELDS AND ITS SOLUTION}

The purpose of this paper is to calculate the greybody factors for the emission of fermion and gauge fields in the background of the metric on their four-dimensional brane induced from a $(4+n)$-dimensional Schwarzschild-like black hole centered on the brane. The starting point of our analysis will be the general equation for the propagation of fields with spin $s=1 / 2$ and 1 in an arbitrary four-dimensional spherically symmetric background. Using the NewmanPenrose formalism [22] (see [23] for a compact review), this general field equation may be written as

$$
\nabla_{A A^{\prime}} \psi^{A B_{1} \cdots B_{2 s-1}=0}
$$

Following the analysis of Cvetic and Larsen [23], it is possible to derive from this general equation a set of $2 s$ differential equations for the components of the wave function of the spin-s field. Specifically, assuming a general, spherically symmetric background of the form 


$$
d s^{2}=-\frac{\Delta}{\Sigma} d t^{2}+\frac{\Sigma}{\Delta} d r^{2}+\Sigma^{2}\left(d \theta^{2}+\sin ^{2} \theta d \varphi^{2}\right)
$$

and using a factorized ansatz for each component of the wave function of the field of the form

$$
\psi_{k-s}=\Delta^{-k / 2}(2 \Sigma)^{k / 2-s} R_{k-s}(r) S_{k-s}(\Omega),
$$

the set of coupled differential equations separates into two sets of decoupled equations for the radial and angular parts of the wave function. In the above expression, $k=0, \ldots, 2 s$ labels the helicity of the components and $S_{k-s}(\Omega)$ $=e^{i m \varphi} d_{k m}^{(j)}(\theta)$, where $j$ and $m$ are the total angular momentum number and its projection on a fixed axis, respectively, and $d_{k m}^{(j)}$ stand for the rotation matrices. Focusing on the radial part of the wave function, the form of the corresponding equation turns out to depend on the helicity of the component. For example, for the upper component $\psi_{s}(k=2 s)$, it takes the form [23]

$$
\begin{aligned}
& \Delta^{s} \frac{d}{d r}\left[\Delta^{1-s} \frac{d R_{s}}{d r}\right]+\left\{\frac{\Sigma^{2} \omega^{2}-i s \omega \Sigma \partial_{r} \Delta}{\Delta}+2 i s \omega \partial_{r} \Sigma-\Lambda\right. \\
& +\Delta\left(s-\frac{1}{2}\right)\left[\partial_{r}\left(\frac{\partial_{r} \Sigma}{\Sigma}\right)+\left(s-\frac{1}{2}\right)\left(\frac{\partial_{r} \Sigma}{\Sigma}\right)^{2}\right. \\
& \left.\left.\quad+(1-s) \frac{\partial_{r} \Sigma}{\Sigma} \frac{\partial_{r} \Delta}{\Delta}\right]\right\} R_{s}=0 .
\end{aligned}
$$

In the above, $\Lambda \equiv j(j+1)-s(s-1)$. As we will shortly see, solving the above equation for the upper component of the field only is sufficient to lead to the determination of the absorption cross section and, thus, the greybody factor.

In the presence of additional spacelike dimensions, the higher-dimensional spherically symmetric generalizations of the four-dimensional Schwarzschild solution have a line element given by

$$
d s^{2}=-h(r) d t^{2}+h(r)^{-1} d r^{2}+r^{2} d \Omega_{2+n}^{2},
$$

where

$$
h(r)=1-\left(\frac{r_{H}}{r}\right)^{n+1}
$$

The angular part of the above higher-dimensional metric tensor is

$$
\begin{aligned}
d \Omega_{2+n}^{2}= & d \theta_{n+1}^{2}+\sin ^{2} \theta_{n+1}\left\{d \theta_{n}^{2}+\sin ^{2} \theta_{n}\left[\cdots+\sin ^{2} \theta_{2}\left(d \theta_{1}^{2}\right.\right.\right. \\
& \left.\left.\left.+\sin ^{2} \theta_{1} d \varphi^{2}\right) \cdots\right]\right\},
\end{aligned}
$$

with $0<\varphi<2 \pi$ and $0<\theta_{i}<\pi$, for $i=1, \ldots, n+1$. It is easy to check that, at distances $r \gg R$, where $R$ is the size of the extra compact dimensions, the above metric tensor reduces to the usual four-dimensional Schwarzschild solution.

Under the assumption that the standard model fields, both fermions and gauge bosons, are restricted to live on a fourdimensional brane, they propagate in a gravitational background which is given by the induced 4D metric on the brane following from the higher-dimensional black hole background (7). This follows if we set $\theta_{i}=\pi / 2$, for $i \geqslant 2$. Specifically, it takes the form

$$
d s^{2}=-h(r) d t^{2}+h(r)^{-1} d r^{2}+r^{2} d \Omega_{2}^{2} .
$$

Comparing Eqs. (4) and (10), we may easily see that the general metric functions appearing in Eq. (4) are now given by $\Sigma=r^{2}$ and $\Delta=h r^{2}$. Substituting in the general, radial differential equation (6), we obtain the following simplified equation:

$$
\begin{aligned}
& \left(h r^{2}\right)^{s} \frac{d}{d r}\left[\left(h r^{2}\right)^{1-s} \frac{d R_{s}}{d r}\right]+\left\{\frac{\omega^{2} r^{2}}{h}+2 i s \omega r\right. \\
& -\frac{i s \omega r}{h}(n+1)(1-h)-\Lambda \\
& \quad-(2 s-1)(s-1)(n+1)(1-h)\} R_{s}=0 .
\end{aligned}
$$

In the above, we have used the relation

$$
\frac{d h}{d r}=\frac{(n+1)}{r}(1-h),
$$

which follows from the definition, Eq. (8). In this paper, we are going to study the cases of fermion $(s=1 / 2)$ and gaugeboson fields $(s=1)$. For those fields, the last term appearing inside the curly brackets in Eq. (11) vanishes independently of the number of projected extra dimensions and, therefore, it will be ignored in the following analysis.

In order to compute the greybody factor for the Hawking radiation in the gravitational background described in Eq. (10), through the emission of fermion and gauge-boson fields, we need to solve Eq. (11) for the radial part of the emitted field in the region outside the horizon of the black hole and all the way to infinity. Because of the complexity of the differential equation, the exact solution appears impossible to derive. As in $[24,13]$ (see also [25] for related works) we are going to follow an approximation method that is suitable for low energies $\omega r_{H} \ll 1$. The method involves solving Eq. (11) first in the vicinity of the black hole, then at infinite distance from it, and, finally, matching the two asymptotic solutions in an intermediate regime. This method will help us derive the form of the absorption coefficient in the equivalent scattering problem of an incoming wave propagating in the background (10), in terms of which the absorption cross section, and thus the greybody factor of the emitted radiation is defined.

As we will shortly see, different components of the emitted field carry different parts of the particle wave function. For an emitted field with spin $s \neq 0$, it is only the upper, $\psi_{s}$, and lower, $\psi_{-s}$, components that are the radiative ones. The upper component will turn out to correspond mainly to the incoming wave while the lower component corresponds mainly to the reflected wave. Moreover, the equation for the lower component is the charge conjugate of the one for the upper component: therefore, solving for any one of them provides the solution for the other one. Here, we will con- 
centrate on the incoming wave and compute the absorption coefficient by comparing the incoming flux at the asymptotic regimes of the horizon and infinity. Matching the two solutions in the intermediate regime, however, is still necessary. We impose the following normalizations for the incoming mode of a field, with spin $s$,

$$
R_{s}^{(h)}=A_{s}^{(h)} h^{-i \beta_{\mathrm{BH}} \omega / 4 \pi},
$$

at the horizon of the black hole, where $\beta_{\mathrm{BH}}=1 / T_{\mathrm{BH}}$, and

$$
R_{s}^{(\infty)}=A_{s}^{(\infty)}(2 \omega r)^{2 s-1} e^{-i \omega r},
$$

at infinity. In the forthcoming sections, and for the solution of the general, radial equation (11), we closely follow the analysis presented in Ref. [13] for the case of the emission of scalar fields.

\section{A. Solving the general equation in the near-horizon zone}

In the vicinity of the black hole, Eq. (11) can be solved by making a change of variable, $r \rightarrow h$. Using Eqs. (8) and (12), we may write the general-spin field equation in the form

$$
\begin{aligned}
h(1- & h) \frac{d^{2} R}{d h^{2}}+\left[(1-s)(1-h)-\frac{(n+2 s)}{(n+1)} h\right] \frac{d R}{d h} \\
& +\left[\frac{\left(\omega r_{H}\right)^{2}}{(n+1)^{2} h(1-h)}+\frac{2 i s \omega r_{H}-\Lambda}{(n+1)^{2}(1-h)}-\frac{i s \omega r_{H}}{(n+1) h}\right] R \\
& =0,
\end{aligned}
$$

where, for simplicity of the notation, the subscript $s$ has been dropped from $R$. By using the redefinition $R(h)=h^{\alpha}(1$ $-h)^{\beta} F(h)$, the above equation takes the form of a hypergeometric equation

$$
h(1-h) \frac{d^{2} F}{d h^{2}}+[c-(1+a+b) h] \frac{d F}{d h}-a b F=0,
$$

with

$$
a=\alpha+\beta+\frac{s+n(1-s)}{(n+1)}, \quad b=\alpha+\beta, \quad c=1-s+2 \alpha .
$$

The power coefficients $\alpha$ and $\beta$, in turn, are found by solving second-order algebraic equations, leading to the results

$$
\alpha_{+}=s+\frac{i \omega r_{H}}{n+1}, \quad \alpha_{-}=-\frac{i \omega r_{H}}{n+1},
$$

and

$$
\beta_{ \pm}=\frac{1}{2(n+1)}\left[1-2 s \pm \sqrt{(1+2 j)^{2}-4 \omega^{2} r_{H}^{2}-8 i s \omega r_{H}}\right]
$$

respectively. The general solution of the hypergeometric equation (16) is

$$
\begin{aligned}
R_{\mathrm{NH}}(h)= & A_{-} h^{\alpha}(1-h)^{\beta} F(a, b, c ; h) \\
& +A_{+} h^{-\alpha}(1-h)^{\beta} F(a-c+1, b-c+1,2-c ; h),
\end{aligned}
$$

where $A_{ \pm}$are arbitrary constants. Expanding the above solution in the limit $r \rightarrow r_{H}$ (equivalently, $h \rightarrow 0$ ) we obtain the asymptotic behavior

$$
\begin{aligned}
R_{\mathrm{NH}} & \simeq A_{-} h^{\alpha}+A_{+} h^{-\alpha} \\
& =A_{-} h^{s} \exp \left(i \omega r_{H}^{n+2} y\right)+A_{+} h^{-s} \exp \left(-i \omega r_{H}^{n+2} y\right)
\end{aligned}
$$

for $\alpha=\alpha_{+}$and

$$
R_{\mathrm{NH}} \simeq A_{-} \exp \left(-i \omega r_{H}^{n+2} y\right)+A_{+} \exp \left(i \omega r_{H}^{n+2} y\right)
$$

for $\alpha=\alpha_{-}$. In the above, we have used the "tortoise" coordinate $y$ defined as

$$
y=\frac{\ln h(r)}{r_{H}^{n+1}(n+1)} .
$$

The choice $\alpha=\alpha_{+}$leads to a solution with an outgoing wave of zero amplitude at the horizon and an incoming wave with infinite amplitude. This is an irregular solution which must be discarded. On the other hand, the choice of $\alpha=\alpha_{-}$leads to regular incoming and outgoing waves with amplitude unity at the horizon. The boundary condition that only incoming modes are to be found in the region outside the horizon of a black hole forces us also to set $A_{+}=0$. After these choices are made, the solution near the horizon has exactly the normalization of the fields described in Eq. (13), since $\alpha_{-}=-i \omega \beta_{\mathrm{BH}} / 4 \pi$, thus determining the asymptotic normalization constant to be $A_{s}^{(h)}=A_{-}$. Turning to the $\beta$ coefficient, the criterion for the convergence of the hypergeometric function $F(a, b, c ; h)$,

$$
\begin{aligned}
& \operatorname{Re}(c-a-b) \\
& \quad=-\left( \pm \frac{1}{n+1} \sqrt{(1+2 j)^{2}-4 \omega^{2} r_{H}^{2}-8 i s \omega r_{H}}\right)>0,
\end{aligned}
$$

clearly demands that we choose $\beta=\beta_{-}$.

We finally need to shift the hypergeometric function towards large values of $r$. This may be done by using a standard linear transformation formula [26], in the following way: 


$$
\begin{aligned}
& R_{\mathrm{NH}}(h)=A_{-} h^{\alpha}\left[\frac{\Gamma(1-s+2 \alpha) \Gamma\left(-2 \beta+\frac{1-2 s}{n+1}\right)}{(1-h)^{\beta} \frac{}{\Gamma(\alpha-\beta+1-s) \Gamma\left(\alpha-\beta+\frac{1-2 s}{n+1}\right)} F(a, b, a+b-c+1 ; 1-h)+(1-h)^{-\beta+(1-2 s) /(n+1)}}\right. \\
&\left.\times \frac{\Gamma(1-s+2 \alpha) \Gamma\left(2 \beta-\frac{1-2 s}{n+1}\right)}{\Gamma(\alpha+\beta) \Gamma\left(\alpha+\beta+\frac{s+n(1-s)}{n+1}\right)} F(c-a, c-b, c-a-b+1 ; 1-h)\right] .
\end{aligned}
$$

We can now "stretch" the above expression towards the intermediate regime, by expanding in the limit $r \rightarrow \infty$, or $h \rightarrow 1$, and take

$$
R_{\mathrm{NH}}(h) \simeq A_{-}\left(\frac{r_{H}}{r}\right)^{-(j+s)} \frac{\Gamma(1-s+2 \alpha) \Gamma\left(-2 \beta+\frac{1-2 s}{n+1}\right)}{\Gamma(\alpha-\beta+1-s) \Gamma\left(\alpha-\beta+\frac{1-2 s}{n+1}\right)}+A_{-}\left(\frac{r_{H}}{r}\right)^{j-s+1} \frac{\Gamma(1-s+2 \alpha) \Gamma\left(2 \beta-\frac{1-2 s}{n+1}\right)}{\Gamma(\alpha+\beta) \Gamma\left(\alpha+\beta+\frac{s+n(1-s)}{n+1}\right)} .
$$

Note that, in order to simplify the procedure of the "matching," the low-energy limit has been taken in the expression of the $\beta$ coefficient in the power of $r$. No expansion has been made, up to this point, in the arguments of the $\Gamma$ functions.

\section{B. Solving the general equation in the far-field zone}

We now need to find the far-field zone solution before being able to match the two asymptotics in the intermediate regime. Going back to the general equation (11) and in the limit $r \rightarrow \infty$ or $h \rightarrow 1$, we obtain

$$
\frac{d^{2} R}{d r^{2}}+\frac{2(1-s)}{r} \frac{d R}{d r}+\left(\omega^{2}+\frac{2 i s \omega}{r}-\frac{\Lambda}{r^{2}}\right) R=0 .
$$

In order to solve the above equation, we set $R$ $=e^{-i \omega r} r^{j+s} \widetilde{R}(r)$. By also making a change of variable $z$ $=2 i \omega r$, the above equation adopts the form of a confluent hypergeometric equation

$$
z \frac{d^{2} \widetilde{R}}{d z^{2}}+(b-z) \frac{d \widetilde{R}}{d z}-a \widetilde{R}=0,
$$

with $a=j-s+1$ and $b=2 j+2$ and the general solution

$$
\widetilde{R}(z)=B_{+} M(a, b, z)+B_{-} U(a, b, z),
$$

where $M$ and $U$ are the Kummer functions and $B_{ \pm}$are arbitrary coefficients. We may therefore write the complete solution for the radial function at infinity as

$$
\begin{aligned}
R_{\mathrm{FF}}(r)= & e^{-i \omega r} r^{j+s}\left[B_{+} M(j-s+1,2 j+2,2 i \omega r)\right. \\
& \left.+B_{-} U(j-s+1,2 j+2,2 i \omega r)\right] .
\end{aligned}
$$

We first need to expand the above expression for large values of $r$. This expansion will help us make sure that the emitted fields have the far-field normalization given in Eq. (14) and will enable us to compute the incoming flux at infinity. In the limit $r \rightarrow \infty$, we find

$$
\begin{aligned}
R_{\mathrm{FF}}(r)= & \frac{e^{-i \omega r} r^{2 s-1}}{(2 i \omega)^{j-s+1}}\left[B_{-}+\frac{B_{+} e^{i \pi(j-s+1)} \Gamma(2 j+2)}{\Gamma(j+s+1)}\right] \\
& +\frac{e^{i \omega r} B_{+} \Gamma(2 j+2)}{\Gamma(j-s+1)(2 i \omega)^{j+s+1} r}+\cdots .
\end{aligned}
$$

The first part of the above expression gives the incoming wave at infinity and, thus, by comparison with Eq. (14), defines the coefficient $A_{s}^{(\infty)}$. The second part gives the outgoing wave. This outgoing part is suppressed as it should be. We remind the reader that the above solution corresponds only to the upper component of the emitted field (either to $\psi_{1 / 2}$ or to $\psi_{1}$ ) which in turn corresponds to the incoming wave. The charge conjugate of the above solution gives the lower component (the other radiative component) of the emitted field, which will have a dominant outgoing wave and a suppressed incoming one.

We may now stretch the far-field solution (30) towards small values of $r$. In the limit $\omega r \ll 1$, we therefore obtain

$$
R_{\mathrm{FF}}(r)=B_{+} r^{j+s}+\frac{B_{-}}{r^{j-s+1}} \frac{\Gamma(2 j+1)}{\Gamma(j-s+1)(2 i \omega)^{2 j+1}} .
$$

Matching the two solutions (26) and (32) in the intermediate regime, we obtain the relations 


$$
\begin{aligned}
& B_{+}=\frac{A_{-}}{r_{H}^{j+s}} \frac{\Gamma(1-s+2 \alpha) \Gamma\left(-2 \beta+\frac{1-2 s}{n+1}\right)}{\Gamma(\alpha-\beta+1-s) \Gamma\left(\alpha-\beta+\frac{1-2 s}{n+1}\right)}, \\
& B_{-}=\frac{A_{-} r_{H}^{j-s+1}(2 i \omega)^{2 j+1} \Gamma(1-s+2 \alpha) \Gamma\left(2 \beta-\frac{1-2 s}{n+1}\right) \Gamma(j-s+1)}{\Gamma(\alpha+\beta) \Gamma\left(\alpha+\beta+\frac{s+n(1-s)}{n+1}\right) \Gamma(2 j+1)} .
\end{aligned}
$$

With the determination of the above arbitrary coefficients, we have now completed the determination of the complete solution for both fermions and gauge-boson fields propagating in the background of a projected $(4+n)$-dimensional Schwarzschild black hole on a four-dimensional brane. We now proceed to the calculation of the greybody factor in each case for an arbitrary number of extra compact spacelike dimensions.

\section{GREYBODY FACTORS FOR THE EMISSION OF FERMIONIC AND GAUGE FIELDS}

As we will shortly see, the expression of the greybody factors for Hawking radiation for fermions and gauge fields involves the quantity $\left|A^{(h)} / A^{(\infty)}\right|^{2}$ determined by the two normalization coefficients appearing in the asymptotic solutions (13) and (14). By using the relations (33), (34), this takes the form

$$
\begin{aligned}
\frac{A^{(h)}}{A^{(\infty)}} & =\frac{A_{-}(2 \omega)^{j+s} e^{i \pi(j-s+1) / 2}}{\left[B_{-}+\frac{B_{+} e^{i \pi(j-s+1)} \Gamma(2 j+2)}{\Gamma(j+s+1)}\right]} \\
& =\frac{\left(2 \omega r_{H}\right)^{j+s} e^{-i \pi(j-s+1) / 2}}{\Gamma(1-s+2 \alpha)\left[C\left(\omega r_{H}\right)^{2 j+1}+D\right]},
\end{aligned}
$$

where the coefficients $C$ and $D$ stand for

$$
\begin{gathered}
C=\frac{2^{2 j+1} e^{i \pi(s-1 / 2)} \Gamma\left(2 \beta-\frac{1-2 s}{n+1}\right) \Gamma(j-s+1)}{\Gamma(\alpha+\beta) \Gamma\left(\alpha+\beta+\frac{s+n(1-s)}{n+1}\right) \Gamma(2 j+1)}, \text { (36 } \\
D=\frac{\Gamma(2 j+2) \Gamma\left(-2 \beta+\frac{1-2 s}{n+1}\right)}{\Gamma(\alpha-\beta+1-s) \Gamma\left(\alpha-\beta+\frac{1-2 s}{n+1}\right) \Gamma(j+s+1)}
\end{gathered}
$$

The measure squared of the ratio $A^{(h)} / A^{(\infty)}$ can then be written as

$$
\left|\frac{A^{(h)}}{A^{(\infty)}}\right|^{2}=\frac{\left(2 \omega r_{H}\right)^{2(j+s)}}{|\Gamma(1-s+2 \alpha)|^{2}\left[C C^{*}\left(\omega r_{H}\right)^{4 j+2}+\left(\omega r_{H}\right)^{2 j+1}\left(C D^{*}+C^{*} D\right)+D D^{*}\right]} .
$$

The derivation of a final explicit result for the greybody factors or emission rates from the above expression requires the evaluation of $\Gamma$ functions for complex arguments. This task is a tedious procedure with the result being highly complicated and unilluminating. Accurate evaluations of the greybody factors and associated emission rates do require use of the above expressions, and so in Sec. IV, we present numerical results for the final expression of the greybody factors, as a function of $\omega r_{H}$, by using the exact value of the above ratio. However, for comparison with previous studies and as in the case of the analysis for the emission of scalar fields [13], a simplified expression may be derived in the lowenergy limit $\omega r_{H} \ll 1$. Expanding the $\Gamma$ functions in this limit, we may express the denominator of the fraction appearing in
Eq. (38) in a power series in $\left(\omega r_{H}\right)$, keeping only the leading term. The result of the expansion depends strongly on the spin of the emitted particle: therefore, we will now discuss separately the results for each case. We emphasize that applications in which accurate values of the emissions rate are required should not use these simplified expressions valid only for $\omega r_{H} \ll 1$, but rather should use the full semiclassical results derived above [together with Eqs. (40), (46), and (49)] as in the evaluation presented in Sec. IV.

\section{A. Spin-1/2 fields}

Following Ref. [23], we define the incoming flux of a fermionic field as the radial component of the conserved cur- 
rent, $J^{\mu}=\sqrt{2} \sigma_{A B}^{\mu} \psi^{A} \bar{\psi}^{B}$, integrated over a two-dimensional sphere. This may be finally written as

$$
\frac{1}{2 \pi} \frac{d N}{d t}=\left|R_{1 / 2}\right|^{2}-\left|R_{-1 / 2}\right|^{2}
$$

in terms of the measure squared of the upper and lower components of the fermionic field. Taking the ratio of the value of this quantity at the horizon with respect to its value at infinity, we obtain the absorption probability for the scattering of an incoming wave traveling from spatial infinity towards the horizon of the projected black hole. It can be simply expressed as

$$
\left|A_{s=1 / 2}\right|^{2}=\left|\frac{A^{(h)}}{A^{(\infty)}}\right|^{2}
$$

if we take into account the fact that the upper component contributes little to the infalling flux both at infinity and at the horizon.

Setting $s=1 / 2$ and expanding the $C$ and $D$ coefficients, defined in Eqs. (36), (37), in the limit $\omega r_{H} \ll 1$, we obtain

$$
\begin{aligned}
C C^{*} & =\frac{2^{-(4 j+2) /(n+1)}}{\Gamma(j+1)^{2}}+\mathcal{O}\left(\omega r_{H}\right)^{2}, \\
C D^{*}+C^{*} D & =\frac{2^{2 j+1}}{\pi}+\mathcal{O}\left(\omega r_{H}\right)^{2}, \\
D D^{*} & =\frac{2^{4 j} 2^{(4 j+2) /(n+1)}}{\pi^{2}} \Gamma(j+1)^{2}+\mathcal{O}\left(\omega r_{H}\right)^{2} .
\end{aligned}
$$

Taking into account the fact that the smallest physically allowed value of the total angular momentum $j$ is the value of the spin, $s=1 / 2$, we easily conclude that the dominant term in the denominator of Eq. (38), in the low-energy limit, is given by the first term in the expansion of $D D^{*}$. Moreover, we have

$\frac{1}{|\Gamma(1 / 2+2 \alpha)|^{2}}=\frac{\cosh (2 \tilde{\alpha} \pi)}{\pi} \simeq \frac{1}{\pi}+\frac{2 \pi\left(\omega r_{H}\right)^{2}}{(n+1)^{2}}+\mathcal{O}\left(\omega r_{H}\right)^{3}$,

where $\widetilde{\alpha}$ is the imaginary part of $\alpha$. Putting everything together, we obtain

$$
\left|A_{s=1 / 2}\right|^{2} \simeq \frac{2 \pi\left(\omega r_{H}\right)^{2 j+1} 2^{-(4 j+2) /(n+1)}}{2^{2 j} \Gamma(j+1)^{2}}+\mathcal{O}\left(\omega r_{H}\right)^{2} .
$$

The absorption probability leads to the absorption cross section $\sigma_{\text {abs }}(\omega)$ via

$$
\sigma_{\text {abs }}^{(s)}(\omega)=\frac{\pi(2 j+1)}{\omega^{2}}\left|A_{s}\right|^{2}
$$

\begin{tabular}{|c|c|c|}
\hline \multirow[t]{2}{*}{$n=2$} & $\begin{array}{l}j=1 / 2 \\
j=3 / 2\end{array}$ & $\begin{array}{l}\sigma_{\mathrm{abs}}^{(1 / 2)} \simeq 2^{5 / 3} \pi r_{H}^{2}+\cdots \\
\sigma_{\mathrm{abs}}^{(1 / 2)} \simeq \frac{2^{4 / 3}}{9} \pi r_{H}^{2}\left(\omega r_{H}\right)^{2}+\cdots\end{array}$ \\
\hline & $j=5 / 2$ & $\sigma_{\mathrm{abs}}^{(1 / 2)} \simeq \frac{\pi}{150} r_{H}^{2}\left(\omega r_{H}\right)^{4}+\cdots$ \\
\hline \multirow[t]{3}{*}{$n=4$} & $j=1 / 2$ & $\sigma_{\mathrm{abs}}^{(1 / 2)} \simeq 2^{1 / 5} 4 \pi r_{H}^{2}+\cdots$ \\
\hline & $j=3 / 2$ & $\sigma_{\mathrm{abs}}^{(1 / 2)} \simeq \frac{4 \pi}{9} 2^{2 / 5} r_{H}^{2}\left(\omega r_{H}\right)^{2}+\cdots$ \\
\hline & $j=5 / 2$ & $\sigma_{\mathrm{abs}}^{(1 / 2)} \simeq \frac{2^{3 / 5}}{75} \pi r_{H}^{2}\left(\omega r_{H}\right)^{4}+\cdots$ \\
\hline \multirow[t]{3}{*}{$n=6$} & $j=1 / 2$ & $\sigma_{\mathrm{abs}}^{(1 / 2)} \simeq 2^{3 / 7} 4 \pi r_{H}^{2}+\cdots$ \\
\hline & $j=3 / 2$ & $\sigma_{\mathrm{abs}}^{(1 / 2)} \simeq \frac{4}{9} 2^{6 / 7} \pi r_{H}^{2}\left(\omega r_{H}\right)^{2}+\cdots$ \\
\hline & $j=5 / 2$ & $\sigma_{\mathrm{abs}}^{(1 / 2)} \simeq \frac{2}{75} 2^{2 / 7} \pi r_{H}^{2}\left(\omega r_{H}\right)^{4}+\cdots$ \\
\hline
\end{tabular}

for any spin $s$ and angular momentum partial wave number $j$. Thus, for the fermion case, the final absorption cross section is, in leading order in $\left(\omega r_{H}\right)$,
TABLE I. Absorption cross section for a brane-localized fermionic field.

$$
\sigma_{\text {abs }}^{(1 / 2)}(\omega)=\frac{2 \pi^{2} r_{H}^{2}(2 j+1)\left(\omega r_{H}\right)^{2 j-1} 2^{-(4 j+2) /(n+1)}}{2^{2 j} \Gamma(j+1)^{2}} .
$$

This is the primary analytic result valid for $\omega r_{H} \ll 1$ for the case of spin-1/2 fermions.

As is obvious from the above formula, the absorption cross section has a strong dependence on the total angular momentum number $j$ of the emitted field as it affects both the power of the dominant $\left(\omega r_{H}\right)$ term and the multiplicative coefficient in front. On the other hand, as in the case of the emission of brane-localized scalar fields studied in [13], no dependence of the power of $\left(\omega r_{H}\right)$ on the number of extra dimensions emerges for a brane-localized fermionic field. Nevertheless, the multiplicative coefficient in front depends on the number of extra dimensions.

In order to be able to draw concrete conclusions about the dependence of $\sigma_{\mathrm{abs}}^{(1 / 2)}$ on $j$ and $n$, the values of the absorption cross section for $n=2,4$, and 6 , and $j=\frac{1}{2}, \frac{3}{2}$, and $\frac{5}{2}$ have been computed and displayed in Table I. By looking at the entries of this table for $j=\frac{1}{2}$ and for every $n$, we see that the absorption cross section becomes proportional to the area of the horizon of the black hole times a numerical coefficient and is independent of the energy of the emitted particle. As the total angular momentum number $j$ increases, the absorption cross section rapidly decreases as it gets suppressed by extra powers of $\omega r_{H}$ and its numerical coefficient also gets smaller. If instead we fix $j$ and vary the number of extra dimensions, then we see that the absorption cross section gets enhanced as the number of the projected extra dimensions increases. The behavior, therefore, of the leading term in the expression of the greybody factor, in the low-energy regime, for the emission of brane fermionic fields is similar to the one for the emission of brane scalar fields [13]. 


\section{B. Spin-1 fields}

We now move to the case of gauge fields. In this case, no conserved current may be defined: however, the incoming flux can be expressed as the integral of the flow of energy, $T^{\mu \nu}=2 \sigma_{A A^{\prime}}^{\mu} \sigma_{B B^{\prime}}^{\nu} \psi^{A B} \bar{\psi}^{A^{\prime} B^{\prime}}$, through a two-dimensional sphere [23]. Then we obtain the expression

$$
\frac{1}{2 \pi} \frac{d N}{d t}=\frac{1}{2 \Sigma \omega}\left(\left|R_{1}\right|^{2}-\left|R_{-1}\right|^{2}\right)
$$

where the redshift of the local energy of photons has been taken into account. The absorption probability is given again by the ratio of the value of the above quantity at the horizon with respect to its value at infinity, and it is found to be

$$
\left|A_{s=1}\right|^{2}=\frac{1}{\left(2 \omega r_{H}\right)^{2}}\left|\frac{A^{(h)}}{A^{(\infty)}}\right|^{2}
$$

Going back to Eq. (38) and setting $s=1$, we expand the $C$ and $D$ coefficients in the limit $\omega r_{H} \ll 1$. Thus we obtain

$$
\begin{aligned}
& C C^{*}=\frac{2^{4 j+2} \Gamma\left(-\frac{2 j+1}{n+1}\right)^{2} \Gamma(j)^{2}}{\Gamma\left(-\frac{j}{n+1}\right)^{2} \Gamma\left(-\frac{j+1}{n+1}\right)^{2} \Gamma(2 j+1)^{2}}+\mathcal{O}\left(\omega r_{H}\right)^{2} \\
& C D^{*}=\frac{-i 2^{2 j+1}}{\pi(n+1)} \frac{\sin \left(\frac{\pi j}{n+1}\right) \sin \left(\frac{\pi(j+1)}{n+1}\right)}{\sin \left(\frac{\pi(2 j+1)}{n+1}\right)}+\mathcal{O}\left(\omega r_{H}\right) \\
& D D^{*}=\frac{\Gamma\left(\frac{2 j+1}{n+1}\right)^{2} \Gamma(2 j+2)^{2}}{\Gamma\left(\frac{j}{n+1}\right)^{2} \Gamma\left(\frac{j+1}{n+1}\right)^{2} \Gamma(j+2)^{2}}+\mathcal{O}\left(\omega r_{H}\right)^{2}
\end{aligned}
$$

The first term in the expansion of $D D^{*}$ gives again the dominant term in the denominator of Eq. (38) in the lowenergy limit. Also, we have

$$
\frac{1}{\Gamma(2 \alpha) \Gamma(-2 \alpha)}=\frac{2 \widetilde{\alpha} \sinh (2 \widetilde{\alpha} \pi)}{\pi} \simeq \frac{4\left(\omega r_{H}\right)^{2}}{(n+1)^{2}}+\mathcal{O}\left(\omega r_{H}\right)^{4}
$$

By using the above results, the absorption probability may be written as

$$
\begin{aligned}
\left|A_{s=1}\right|^{2} \simeq & \frac{4\left(\omega r_{H}\right)^{2}\left(2 \omega r_{H}\right)^{2 j}}{(n+1)^{2}} \frac{\Gamma\left(\frac{j}{n+1}\right)^{2} \Gamma\left(\frac{j+1}{n+1}\right)^{2} \Gamma(j+2)^{2}}{\Gamma\left(\frac{2 j+1}{n+1}\right)^{2} \Gamma(2 j+2)^{2}} \\
& +\mathcal{O}\left(\omega r_{H}\right)^{2}
\end{aligned}
$$

TABLE II. Absorption cross section for a brane-localized gauge field.

$$
\begin{array}{rlrl}
\hline n=2 & j=1 & \sigma_{\mathrm{abs}}^{(1)} & \simeq \frac{64}{81} \pi^{3} r_{H}^{2}\left(\omega r_{H}\right)^{2}+\cdots \\
& j=2 & \sigma_{\mathrm{abs}}^{(1)} & \simeq \frac{\pi}{5} r_{H}^{2}\left(\omega r_{H}\right)^{4}+\cdots \\
& j=3 & \sigma_{\mathrm{abs}}^{(1)} & \simeq \frac{4 \pi}{1575} r_{H}^{2}\left(\omega r_{H}\right)^{6}+\cdots \\
n=4 & j=1 & \sigma_{\mathrm{abs}}^{(1)} & \simeq \frac{4}{75} 2^{2 / 5} \Gamma\left(\frac{1}{10}\right)^{2} \Gamma\left(\frac{2}{5}\right)^{2} r_{H}^{2}\left(\omega r_{H}\right)^{2}+\cdots \\
& j=2 & \sigma_{\mathrm{abs}}^{(1)} & \simeq \frac{4 \pi}{125} \Gamma\left(\frac{2}{5}\right)^{2} \Gamma\left(\frac{3}{5}\right)^{2} r_{H}^{2}\left(\omega r_{H}\right)^{4}+\cdots \\
& j=3 & \sigma_{\mathrm{abs}}^{(1)} & \simeq \frac{64 \pi}{39375} \frac{\Gamma(3 / 5)^{2} \Gamma(4 / 5)^{2}}{\Gamma(7 / 5)^{2}} r_{H}^{2}\left(\omega r_{H}\right)^{6}+\cdots \\
n=6 & j=1 & \sigma_{\mathrm{abs}}^{(1)} & \simeq \frac{16 \pi}{147} \frac{\Gamma(1 / 7)^{2} \Gamma(2 / 7)^{2}}{\Gamma(3 / 7)^{2}} r_{H}^{2}\left(\omega r_{H}\right)^{2}+\cdots \\
& j=2 & \sigma_{\mathrm{abs}}^{(1)} & \simeq \frac{2^{6 / 7}}{245} \Gamma\left(\frac{3}{14}\right)^{2} \Gamma\left(\frac{2}{7}\right)^{2} r_{H}^{2}\left(\omega r_{H}\right)^{4}+\cdots \\
& j=3 & \sigma_{\mathrm{abs}}^{(1)} & \simeq \frac{64}{77175} \frac{\pi^{3}}{\sin ^{2}(4 \pi / 7)} r_{H}^{2}\left(\omega r_{H}\right)^{6}+\cdots \\
& & & \\
& j=\cdots
\end{array}
$$

leading to the following absorption cross section, for the gauge field case, in leading order in $\omega r_{H}$ :

$$
\begin{aligned}
\sigma_{\mathrm{abs}}^{(1)}(\omega)= & 4 \pi r_{H}^{2}\left(2 \omega r_{H}\right)^{2 j} \frac{(2 j+1)}{(n+1)^{2}} \\
& \times\left(\frac{\Gamma\left(\frac{j}{n+1}\right) \Gamma\left(\frac{j+1}{n+1}\right) \Gamma(j+2)}{\Gamma\left(\frac{2 j+1}{n+1}\right) \Gamma(2 j+2)}\right)^{2}
\end{aligned}
$$

This is our primary analytic result valid for $\omega r_{H} \ll 1$ for the case of gauge fields.

As in the case of scalar and fermionic fields, the absorption cross section for brane-localized gauge fields depends nontrivially on the angular momentum number $j$ and the number of extra dimensions, $n$. The power of the dominant $\left(\omega r_{H}\right)$ term depends only on $j$, but the numerical coefficient in front depends strongly on both parameters. The values of the above quantity for $n=2,4$, and 6 and $j=1,2$, and 3 are given in Table II.

Unlike the case of scalar and fermionic field emission, the absorption cross section for the emission of gauge fields depends on the energy of the emitted particle even in the case of the lowest partial wave with $j=1$. This follows from the different dependence of the dominant $\left(\omega r_{H}\right)$ term on the angular momentum number. Any further increase in the value of $j$ leads to the appearance of a suppression factor of powers of $\left(\omega r_{H}\right)$, while the increase in the number of extra dimensions strongly enhances the absorption cross section. 

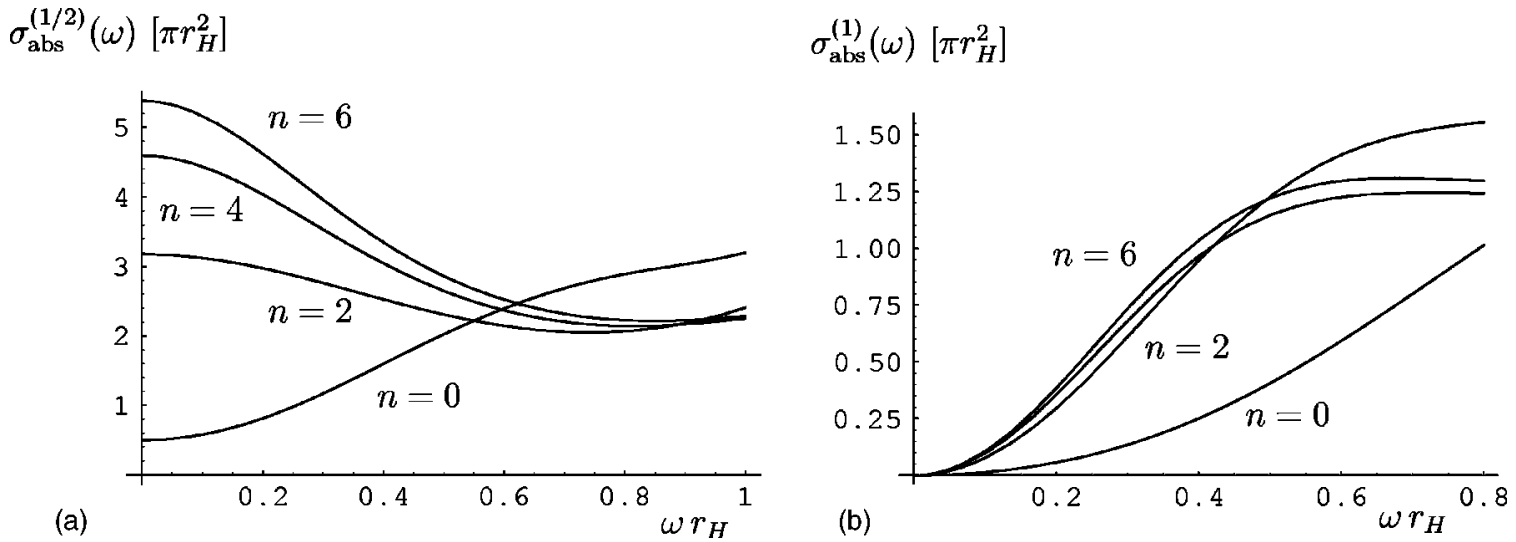

FIG. 1. Greybody factors $\sigma_{\text {abs }}(\omega)$ for the emission of (a) fermions and (b) gauge brane-localized fields, as a function of $\omega r_{H}$, for $n$ $=0,2,4$, and 6 .

\section{EVALUATION OF RESULTS}

In this section, we proceed to calculate the corresponding emission rates for spin-1/2 and -1 fields in the background of the projected four-dimensional black hole given in Eq. (10). In this section we will not use the simplified expressions valid only for $\omega r_{H} \ll 1$, but rather the full analytic expressions, Eqs. (36) -(38).

For the relevant four-dimensional process of the emission of brane-localized fields, Eq. (2) takes the form

$$
\frac{d E(\omega)}{d t}=\sum_{j} \sigma_{j}(\omega) \frac{\omega^{3}}{\exp \left(\omega / T_{\mathrm{BH}}\right) \mp 1} \frac{d \omega}{2 \pi^{2}} .
$$

Thus, for the evaluation of the emission rates, we need the expressions of the greybody factors summed over the angular momentum number $j$. A simple numerical analysis shows that, by summing over the first three partial waves, we obtain the dependence of the greybody factors on the parameter $\omega r_{H}$ to high accuracy, with all higher partial waves adding an almost zero contribution to the final result. The numerical evaluation gives us the ability to use the exact value of the ratio (38) instead of the simplified leading-order corrections presented in Eqs. (47) and (55), which are valid only for $\omega r_{H} \ll 1$. Such a numerical analysis will reveal how fast the low-energy approximation breaks down as the expansion parameter $\omega r_{H}$ increases. Figures 1(a) and 1(b) depict the greybody factors for fermions and gauge fields, respectively, in units of $\pi r_{H}^{2}$, as functions of $\omega r_{H}$. The first three partial waves have been summed in each case, and the dependence for $n=0,2,4$, and 6 is shown. We observe that, for small enough values of $\omega r_{H}$, we reproduce, as expected, the behavior given by the leading-order corrections and according to which the greybody factors are enhanced as the number of extra dimensions projected on our brane increases. This agreement holds for both species of fields up to the value $\omega r_{H} \simeq 0.4$. As $\omega r_{H}$ increases further, it is the lowerdimensional models that seem to give the largest greybody factors. However, our calculation is limited to a WKB-like semiclassical approximation and only an exact numerical analysis of the original master equation, Eq. (11), can determine $\sigma_{\text {abs }}(\omega)$ in the intermediate and high-energy regime $\omega r_{H} \gtrsim 1$. Let us finally note that our analysis correctly reproduces the behavior of the greybody factors in the case of $n$ $=0 \quad[21,27]$, with $\sigma_{\text {abs }}^{(1 / 2)}(\omega)$ adopting a nonvanishing asymptotic value at very low energies and $\sigma_{\text {abs }}^{(1)}(\omega)$ going to zero. The same behavior is observed in the cases with $n$ $\neq 0$.

Figures 2(a) and 2(b) display the behavior of the differential emission rate per time unit and energy interval $d^{2} E / d t d \omega$ versus the parameter $\omega r_{H}$, for fermions and gauge fields, as follows by combining Eq. (56) with the exact expression of the greybody factor in each case and the definition of the temperature of the projected four-dimensional black hole, $T_{H}=(n+1) / 4 \pi r_{H}$. The emission rates are substantially enhanced as the number of extra dimensions increases. The increase is larger for gauge fields which leads to

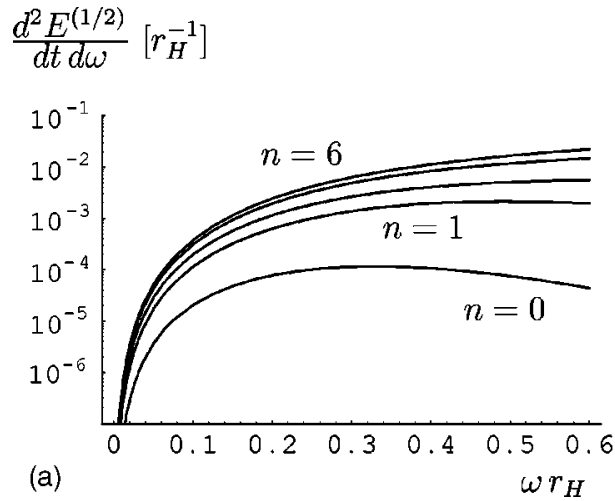

$\frac{d^{2} E^{(1)}}{d t d \omega}\left[r_{H}^{-1}\right]$

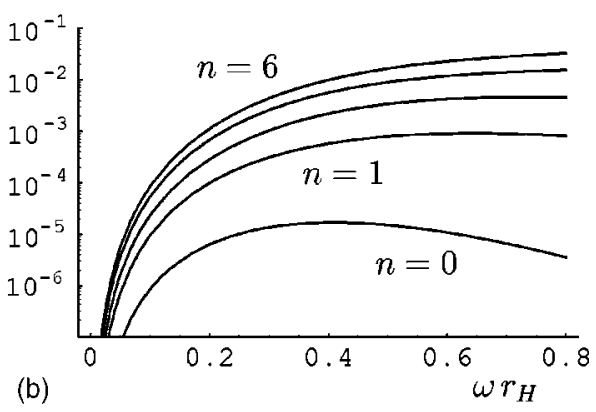

FIG. 2. Differential emission rate $d^{2} E / d t d \omega$ for (a) fermions and (b) gauge fields, as a function of $\omega r_{H}$, for $n=0,1,2,4$, and 6 . 


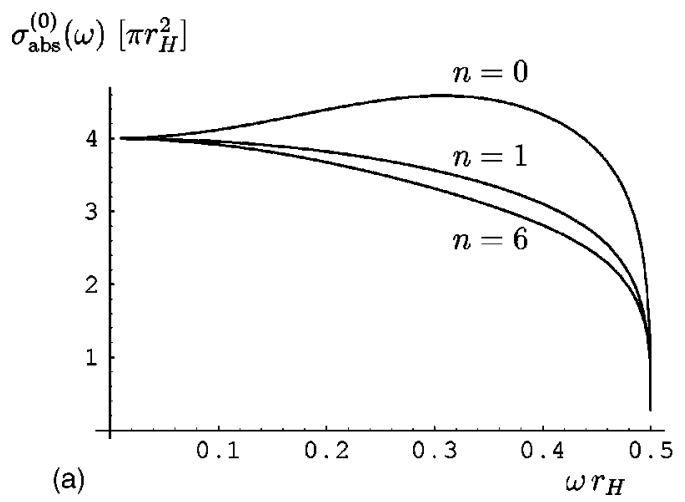

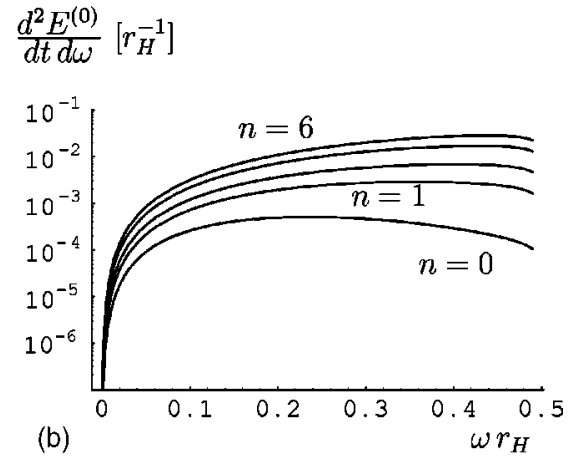

FIG. 3. (a) Greybody factor for the emission of branelocalized scalar fields for $n=0,1$, and 6 ; (b) the corresponding differential emission rate for $n=0,1$, 2,4 , and 6 . the result that the emission rates for the two species become comparable, for large values of $n$. On the other hand, for low values of $n$, the emission rate for a fermion is considerably larger compared than that for a gauge field, with the difference reaching an order of magnitude in the limiting case of $n=0$.

It is useful to perform a similar numerical analysis for the case of scalar fields being emitted by the same fourdimensional black hole, a case studied in Ref. [13]. By following a similar analytical approach, the leading-order correction in the expression of the greybody factor in that case was also derived. According to those results, the leading correction for the first, and thus dominant, partial wave was independent of the number of extra dimensions. Any difference in the value of the greybody factor was to come from the higher partial waves whose coefficient indeed increased with increasing $n$. As $\omega r_{H}$ increases, however, the next-toleading-order corrections for each partial wave (for example, the terms being denoted by ellipses in Tables I and II, and similarly in Table II in [13]) can dominate the contribution coming from the higher partial waves. In the case of fermions and gauge fields, we showed that this does not happen until the value $\omega r_{H} \simeq 0.4$. For scalar fields, it turns out that this value is much smaller. Figure 3(a) depicts the greybody factor for the emission of brane-localized scalar fields, by using the exact value of the ratio of the two coefficients $B_{+}$ and $B_{-}$, given in Eq. (46) of Ref. [13]. We immediately see that, for extremely small values of $\omega r_{H}$, the greybody factors, for various values of $n$, converge to the same asymptotic value, in agreement with our simplified expression. However, very soon, the next-to-leading-order corrections dominate and cause the greybody factor to decrease as $n$ increases. The emission rate for scalar fields is nevertheless enhanced as the number of extra dimensions increases. This is caused by the decrease in the denominator of Eq. (56), due to the dependence of the temperature $T_{\mathrm{BH}}$ on $n$, that overcomes the decrease of the greybody factor. As in the case of fermion and gauge fields, the increase amounts to orders of magnitude compared to the limiting case of $n=0$.

\section{CONCLUSIONS}

In an earlier paper [13], we studied the problem of the decay of a higher-dimensional Schwarzschild-like black hole through the emission of either higher-dimensional bulk, or brane-localized, scalar modes. In this paper, we extended the computation of the greybody factors that appear in the Hawking decay process to the spin-1/2 and spin-1 branelocalized cases of greatest phenomenological interest. Since the angular momentum of the black hole has been ignored, our calculations are relevant to the post-balding and spindown phases of the life of a black hole produced at a highenergy collider or by ultrahigh-energy cosmic-ray interactions. The semiclassical approximation methods employed allow us to calculate the greybody factors and the corresponding emission rates for a general number $n$ of flat extra dimensions of the Arkani-Hamed, Dimopoulos, and Dvali type. (It is important to bear in mind that black holes in highly curved bulk spacetimes-for example, RandallSundrum 5D theories-require a somewhat modified treatment from the one presented here.) The primary analytic results of our calculation for the greybody factors, for arbitrary $j$ and $n$, are presented in Eqs. (35)-(38) and (40), (46), and (49). Simplified expressions are provided in Eqs. (47) and (55) for spin 1/2 and spin 1, respectively. In both cases there is an increase in the greybody factor as $n$ is increased at fixed $j$. As the subsequent numerical analysis revealed, this behavior survives up to intermediate values of the energy of the emitted particle and contributes to a considerably enhanced emission rate for both fermions and gauge bosons. A similar analysis for the emission of scalar fields also led to an enhancement of the emission rate as $n$ increases despite the fact that the greybody factor, rapidly deviating from the behavior dictated by the leading-order correction in the lowenergy approximation, is suppressed. We expect that our results will be of use in a detailed study of the signatures of possible black hole production events.

Note added. While writing this paper, the related work of Ref. [28] appeared which also considers the greybody factors for black holes in brane-world theories.

\section{ACKNOWLEDGMENTS}

We wish to thank Roberto Emparan and Nemanja Kaloper for discussions. 
[1] N. Arkani-Hamed, S. Dimopoulos, and G. R. Dvali, Phys. Lett. B 429, 263 (1998); Phys. Rev. D 59, 086004 (1999); I. Antoniadis, N. Arkani-Hamed, S. Dimopoulos, and G. R. Dvali, Phys. Lett. B 436, 257 (1998).

[2] K. Akama, Lect. Notes Phys. 176, 267 (1982); V. A. Rubakov and M. E. Shaposhnikov, Phys. Lett. 125B, 139 (1983); 125B, 136 (1983); M. Visser, ibid. 159B, 22 (1985); I. Antoniadis, Phys. Lett. B 246, 377 (1990); I. Antoniadis, K. Benakli, and M. Quiros, ibid. 331, 313 (1994); J. D. Lykken, Phys. Rev. D 54, 3693 (1996).

[3] L. Randall and R. Sundrum, Phys. Rev. Lett. 83, 3370 (1999); 83, 4690 (1999).

[4] I. Antoniadis, K. Benakli, and M. Quiros, Phys. Lett. B 460, 176 (1999); N. Arkani-Hamed, S. Dimopoulos, and J. MarchRussell, Phys. Rev. D 63, 064020 (2001); R. Sundrum, ibid. 59, 085009 (1999); G. F. Giudice, R. Rattazzi, and J. D. Wells, Nucl. Phys. B544, 3 (1999); E. A. Mirabelli, M. Perelstein, and M. E. Peskin, Phys. Rev. Lett. 82, 2236 (1999); J. L. Hewett, ibid. 82, 4765 (1999); T. Han, J. D. Lykken, and R. J. Zhang, Phys. Rev. D 59, 105006 (1999); N. Kaloper, J. March-Russell, G. D. Starkman, and M. Trodden, Phys. Rev. Lett. 85, 928 (2000); S. Cullen and M. Perelstein, ibid. 83, 268 (1999); S. Hannestad and G. Raffelt, ibid. 87, 051301 (2001); L. J. Hall and D. R. Smith, Phys. Rev. D 60, 085008 (1999); N. ArkaniHamed, S. Dimopoulos, N. Kaloper, and J. March-Russell, Nucl. Phys. B567, 189 (2000); S. Cullen, M. Perelstein, and M. E. Peskin, Phys. Rev. D 62, 055012 (2000); P. Kanti and K. A. Olive, ibid. 60, 043502 (1999); Phys. Lett. B 464, 192 (1999); A. Dedes and P. Kanti, hep-ph/0003051; R. Guedens, D. Clancy, and A. R. Liddle, Phys. Rev. D 66, 043513 (2002); T. Matsuda, ibid. 66, 107301 (2002); M. Bastero-Gil, V. Di Clemente, and S. F. King, ibid. 67, 083504 (2003).

[5] P. C. Argyres, S. Dimopoulos, and J. March-Russell, Phys. Lett. B 441, 96 (1998).

[6] T. Banks and W. Fischler, hep-th/9906038.

[7] S. B. Giddings and S. Thomas, Phys. Rev. D 65, 056010 (2002).

[8] S. Dimopoulos and G. Landsberg, Phys. Rev. Lett. 87, 161602 (2001).

[9] S. Dimopoulos and R. Emparan, Phys. Lett. B 526, 393 (2002); G. F. Giudice, R. Rattazzi, and J. D. Wells, Nucl. Phys. B630, 293 (2002); D. M. Eardley and S. B. Giddings, Phys. Rev. D 66, 044011 (2002).

[10] M. B. Voloshin, Phys. Lett. B 518, 137 (2001); 524, 376 (2002).

[11] S. Hossenfelder, S. Hofmann, M. Bleicher, and H. Stocker, Phys. Rev. D 66, 101502(R) (2002); Phys. Lett. B 548, 73 (2002); H. C. Kim, S. H. Moon, and J. H. Yee, J. High Energy Phys. 02, 046 (2002); K. Cheung, Phys. Rev. Lett. 88, 221602 (2002); Phys. Rev. D 66, 036007 (2002); R. Casadio and B. Harms, Int. J. Mod. Phys. A 17, 4635 (2002); S. Hofmann, M. Bleicher, L. Gerland, S. Hossenfelder, S. Schwabe, and H. Stocker, hep-ph/0111052; G. Pasztor and T. G. Rizzo, in Proceedings of the APS/DPF/DPB Summer Study on the Future of Particle Physics, Snowmass, 2001, edited by R. Davidson and C. Quigg, hep-ph/0112054; G. Landsberg, Phys. Rev. Lett. 88, 181801 (2002); E. J. Ahn, M. Cavaglia, and A. V. Olinto, Phys. Lett. B 551, 1 (2003); U. Baur et al., in Proceedings of the
APS/DPF/DPB Summer Study on the Future of Particle Physics, Snowmass, 2001, edited by R. Davidson and C. Quigg, hep-ph/0201227; T. G. Rizzo, J. High Energy Phys. 02, 011 (2002); S. N. Solodukhin, Phys. Lett. B 533, 153 (2002); V. Cardoso and J. P. Lemos, ibid. 538, 1 (2002); Phys. Rev. D 67, 084005 (2003); V. Cardoso, O. J. C. Dias, and J. P. S. Lemos, ibid. 67, 064026 (2003); A. Chamblin and G. C. Nayak, ibid. 66, 091901(R) (2002); V. Frolov and D. Stojkovic, ibid. 66, 084002 (2002); Phys. Rev. Lett. 89, 151302 (2002); S. C. Park and H. S. Song, hep-ph/0111069; Y. Uehara, hep-ph/0205068; hep-ph/0205199; A. V. Kotwal and C. Hays, Phys. Rev. D 66, 116005 (2002); T. Han, G. D. Kribs, and B. McElrath, Phys. Rev. Lett. 90, 031601 (2003).

[12] A. Goyal, A. Gupta, and N. Mahajan, Phys. Rev. D 63, 043003 (2001); J. L. Feng and A. D. Shapere, Phys. Rev. Lett. 88, 021303 (2002); L. Anchordoqui and H. Goldberg, Phys. Rev. D 65, 047502 (2002); R. Emparan, M. Masip, and R. Rattazzi, ibid. 65, 064023 (2002); L. A. Anchordoqui, J. L. Feng, H. Goldberg, and A. D. Shapere, ibid. 65, 124027 (2002); Y. Uehara, Prog. Theor. Phys. 107, 621 (2002); J. Alvarez-Muniz, J. L. Feng, F. Halzen, T. Han, and D. Hooper, Phys. Rev. D 65, 124015 (2002); A. Ringwald and H. Tu, Phys. Lett. B 525, 135 (2002); M. Kowalski, A. Ringwald, and H. Tu, ibid. 529, 1 (2002); D. Kazanas and A. Nicolaidis, hep-ph/0109247; P. Jain, S. Kar, S. Panda, and J. P. Ralston, hep-ph/0201232.

[13] P. Kanti and J. March-Russell, Phys. Rev. D 66, 024023 (2002).

[14] N. Arkani-Hamed, S. Dimopoulos, G. R. Dvali, and J. MarchRussell, Phys. Rev. D 65, 024032 (2002); K. R. Dienes, E. Dudas, and T. Gherghetta, Nucl. Phys. B557, 25 (1999).

[15] K. R. Dienes, E. Dudas, and T. Gherghetta, Phys. Lett. B 436, 55 (1998); Nucl. Phys. B537, 47 (1999).

[16] See, e.g., J. Hewett and J. March-Russell, in Review of Particle Properties [Phys. Rev. D 66, 010001 2002].

[17] R. C. Myers and M. J. Perry, Ann. Phys. (N.Y.) 172, 304 (1986).

[18] R. Emparan, J. Garcia-Bellido, and N. Kaloper, J. High Energy Phys. 01, 079 (2003).

[19] R. Emparan, G. T. Horowitz, and R. C. Myers, Phys. Rev. Lett. 85, 499 (2000).

[20] S. W. Hawking, Commun. Math. Phys. 43, 199 (1975).

[21] D. N. Page, Phys. Rev. D 13, 198 (1976); W. G. Unruh, ibid. 14, 3251 (1976); N. Sanchez, ibid. 18, 1030 (1978).

[22] S. Chandrasekhar, The Mathematical Theory of Black Holes (Oxford University Press, New York, 1983).

[23] M. Cvetic and F. Larsen, Phys. Rev. D 57, 6297 (1998).

[24] S. R. Das and S. D. Mathur, Nucl. Phys. B478, 561 (1996); J. Maldacena and A. Strominger, Phys. Rev. D 55, 861 (1997); S. R. Das, G. W. Gibbons, and S. D. Mathur, Phys. Rev. Lett. 78, 417 (1997).

[25] S. S. Gubser, Phys. Rev. D 56, 7854 (1997); R. Emparan, Nucl. Phys. B516, 297 (1998); V. Frolov and D. Stojkovic, Phys. Rev. D 67, 084004 (2003).

[26] Handbook of Mathematical Functions, edited by M. Abramowitz and I. Stegun (Academic, New York, 1966).

[27] J. H. MacGibbon and B. R. Webber, Phys. Rev. D 41, 3052 (1990).

[28] D. Ida, K. y. Oda, and S. C. Park, Phys. Rev. D 67, 064025 (2003). 